\title{
Ergonomics in robotic surgery: patients' safety and protection during complex procedures
}

\author{
Samuel S. Stefan ${ }^{1}$, Yousra Ahmad', Jim S. Khan ${ }^{1,3}$ \\ ${ }^{1}$ Colorectal Department, Queen Alexandra Hospital, Portsmouth PO6 3LY, UK. \\ ${ }^{2}$ Anaesthetics Department, Queen Alexandra Hospital, Portsmouth PO6 3LY, UK. \\ ${ }^{3}$ School of Health, Education, Medicine and Social Care, Anglia Ruskin University, Cambridge CB1 1PT, UK. \\ Correspondence to: Dr. Jim S. Khan, Colorectal Department, Queen Alexandra Hospital, Southwick Hill Road, Cosham, \\ Portsmouth PO6 3LY, UK. E-mail: Jim.Khan@porthosp.nhs.uk
}

How to cite this article: Stefan SS, Ahmad Y, Khan JS. Ergonomics in robotic surgery: patients' safety and protection during complex procedures. Mini-invasive Surg 2021;5:23. https://dx.doi.org/10.20517/2574-1225.2021.24

Received: 23 Feb 2021 First Decision: 12 Mar 2021 Revised: 12 Mar 2021 Accepted: 24 Mar 2021 Published: 8 May 2021

Academic Editor: Simon Ng Copy Editor: Xi-Jun Chen Production Editor: Xi-Jun Chen

\begin{abstract}
Specific injuries due to poor positioning seen in robotic pelvic surgery include slips, compartment syndrome, facial oedema, injuries on pressure points, and accidental injuries caused by the robotic arms. The use of the vacuum bean-bag positioner, L-bar against the patient's face, and inflated gloves for hand support are simple and effective techniques and should be included in the standard operating policies for robotic surgery. We recommend use of the "L" shaped safety bar against the patient's face to ensure protection against accidental injuries caused by the robotic arms. The anti-slip bean-bag mattress is efficient to prevent slipping; it conforms to the shape of the body for stable positioning and allows extremities to lie in a natural position. Protection of pressure points of hands and elbows can be done with inflated medical gloves placed in the patient's hands. Surgeons, anaesthetists and theatre teams are together responsible for ensuring that safety measures are in place to reduce the risk of these complications.
\end{abstract}

Keywords: Robotic surgery, ergonomics, intraoperative injuries, positional complications

The last decade has seen some significant technological advances in surgery, leading to improved outcomes; however, as with every new technique there is an associated learning curve, which often entails longer operating times. The patients are usually placed in steep Trendelenburg and tilted positions in pelvic surgery. It is extremely important to ensure that the patients do not slip off the operating table during these 
procedures and to avoid the risk of injuries. Specific injuries which are due to poor positioning include slips, compartment syndrome, facial oedema, and injuries on pressure points, including peripheral neuropathy ${ }^{[1,2]}$. Incorrect positioning of the upper and lower limbs on the operating table can lead to peripheral nerve injuries (ulnar, peroneal) and brachial plexopathies, due to compression or stretching ${ }^{[1]}$.

Robotic surgery adds another element to this complexity by the presence of the insensate robotic arms, and the operator seated at a distance from the operating field. Accidental injuries caused by the robotic arms can result in bruising, burns due to friction of the robotic arms against the body, and facial injuries with potential dislodgement of the endotracheal tube ${ }^{[3]}$.

The development of a standard operating policy for perioperative care in robotic surgery has been advocated to prevent the above-mentioned complications. Awareness of these potential injuries should be raised and discussed when the patient is consented for the operation, at the team brief meeting and during the robotic procedure ${ }^{[4]}$. Surgeons, anaesthetists and theatre teams are all responsible for ensuring that safety measures are in place to reduce the risk of these complications ${ }^{[5]}$.

The robotic surgical team at our institution has gained experience of over eight years. Working in a multidisciplinary collaboration has enabled us to design a safe pathway for robotic surgery. We have been able to minimise the risk and proceed with safe surgery by following these standardised operating procedures.

We recommend the use of the "L" shaped safety bar above the patient's face [Figure 1]. This bar is fixed to the operating table and covers the patient's face and the endotracheal tube, ensuring protection against accidental injuries caused by the contact with the colliding robotic arms.

Various methods have been reported in literature to prevent the slipping of the patient off the table intraoperatively, including straps, shoulder restraints, leg straps and anti-slip mattresses ${ }^{[2]}$. We have found that the anti-slip bean-bag mattress is an efficient piece of equipment for preventing slipping [Figure 2]. It is also named surgical vacuum bean bag positioner and is used to prevent the movement of the patient during the Trendelenburg positioning and tilted position of the operating table. Once vacuumed, it conforms to the shape of the body for stable positioning and allows the extremities to lie in a natural position.

Secondly, protection of pressure points at the level of the hands and elbows is extremely important, especially during lengthy procedures, to prevent neuropraxia and compartment syndrome in the hand and forearm ${ }^{[4]}$. The use of the inflated medical gloves placed in the hands of the patients is a new technique developed at our institute. The hand and the wrist can rest in a relaxed fashion over these gloves, and the fingers are well supported, too. The thumbs must be kept uppermost. This arrangement can protect the pressure points and prevent neurological injuries [Figure 3].

Lastly, along with these standard procedures, the patient is returned to the supine neutral position for 15 min after every $4 \mathrm{~h}$ to avoid compartment syndrome, and calf compression pumps are preferred over the anti-embolism stockings.

These techniques have been used in our institute for the last 10 years in both laparoscopic and robotic surgery. The local incidence of patient slippage and pressure point injuries was $0.5 \%$. This compares favourably low with the overall incidence of 3.6\% quoted in robotic rectal cancer operations performed in lithotomy ${ }^{[5]}$. 

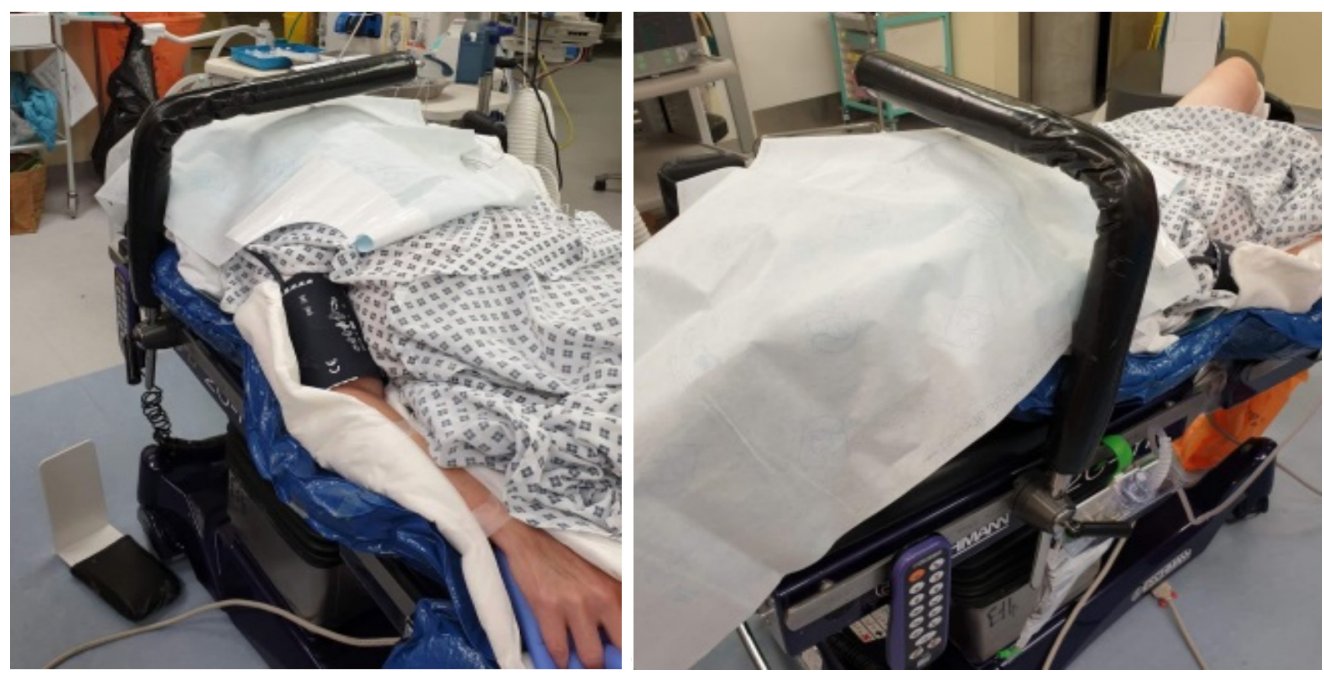

Figure 1. Setup of the "L" protective metallic bar in situ, along with the anti-slip mattress.

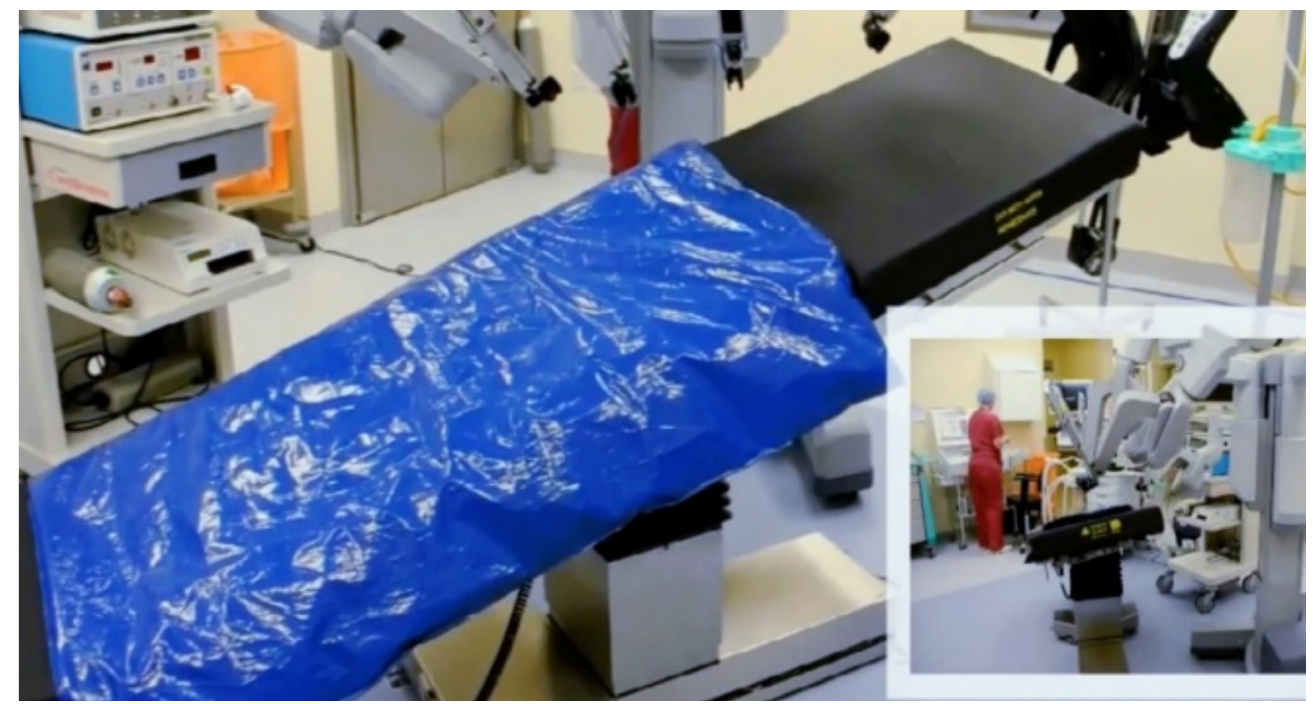

Figure 2. The use of the anti-slip bean-bag mattress.

Every team member involved in the robotic procedure has the responsibility to ensure the patient's safety. Positional complications in surgery although rare, can have serious consequences. As well as causing harm to the patient, there are also potentially increased costs for healthcare and medico-legal implications. Awareness, team training and development of standard operating policies for robotic surgery can reduce the incidence of these perioperative complication $s^{[1,2]}$. The use of vacuum bean-bag positioner, L-bar against the patient's face, and inflated gloves for hand support are simple and effective techniques that are hereby proposed to be included in the standard operating policies for robotic surgery, to prevent positional complications. 

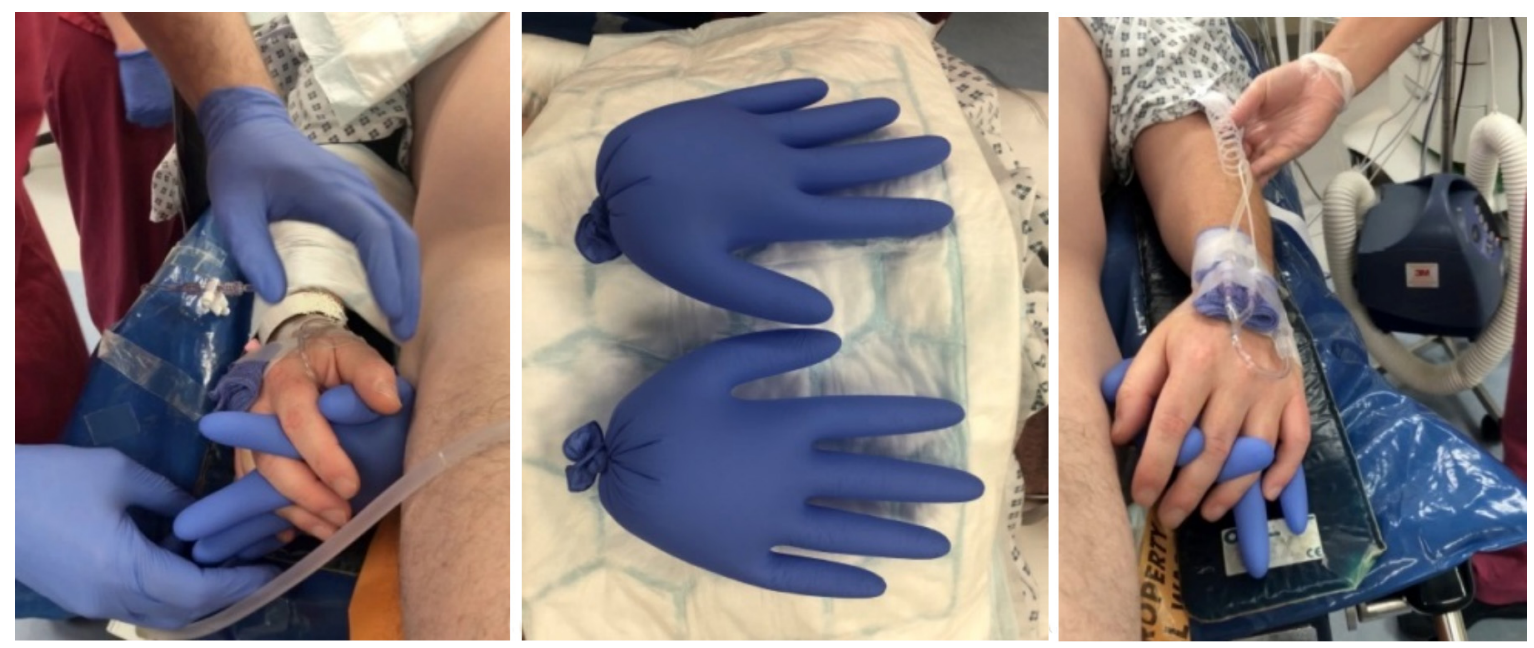

Figure 3. The use of inflated medical gloves to protect the patient's hands and fingers during lengthy surgical procedures.

\section{DECLARATIONS}

\section{Authors' contributions}

Wrote and reviewed the manuscript: Stefan SS, Ahmad Y, Khan JS

\section{Availability of data and materials}

Not applicable.

\section{Financial support and sponsorship}

None.

\section{Conflicts of interest}

All authors declared that there are no conflicts of interest.

\section{Ethical approval and consent to participate}

Not applicable.

\section{Consent for publication}

Not applicable.

\section{Copyright}

(c) The Author(s) 2021.

\section{REFERENCES}

1. Maerz DA, Beck LN, Sim AJ, Gainsburg DM. Complications of robotic-assisted laparoscopic surgery distant from the surgical site. $B r J$ Anaesth 2017;118:492-503. DOI PubMed

2. Waqas A, Arulampalam T, Naqvi S, Khan J. Positional complications of minimal access surgery, laparoscopic/robotic/transanal surgery. Colorectal Dis 2018;20:449-50. DOI PubMed

3. Song JB, Vemana G, Mobley JM, Bhayani SB. The second "time-out": a surgical safety checklist for lengthy robotic surgeries. Patient Saf Surg 2013;7:19. DOI PubMed PMC

4. Codd R, Evans M, Sagar P, Williams GL. A systematic review of peripheral nerve injury following laparoscopic colorectal surgery. Colorectal Dis 2013;15:278-82. DOI PubMed

5. Ross H, Lee SW, Champagne BJ, Pigazzi A, Rivadeneira DE. Robotic approaches to colorectal surgery. Switzerland: Springer International Publishing; 2015. DOI 